مجلة القادسية لعلوم الطب البيطري العدد الخاص ببحوث المؤتمر العلمي الخامس 21-2012/11/22 المجلد 11 العبطري 3012

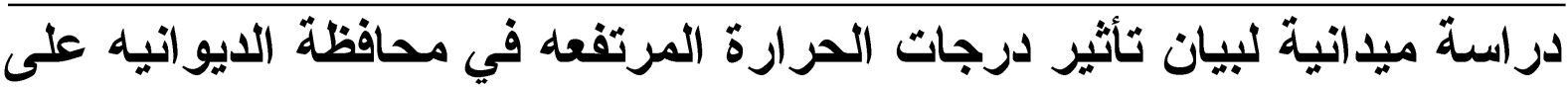

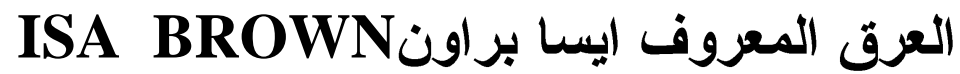

علاء عبد العزيز عبد

\author{
اسر اء نجم عبد الله \\ كلية الطب البيطري/ جامعة القادسية \\ الخلاصة جانة
}

نافع صبيح جاسم

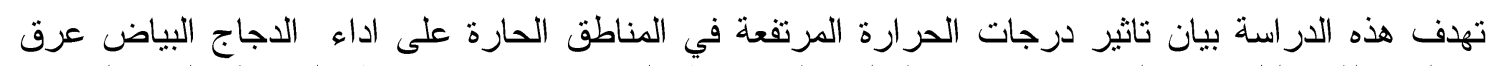

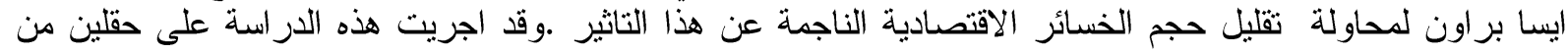

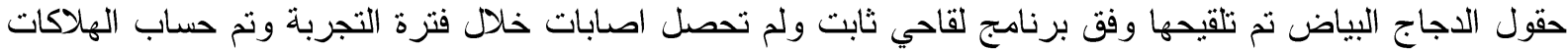

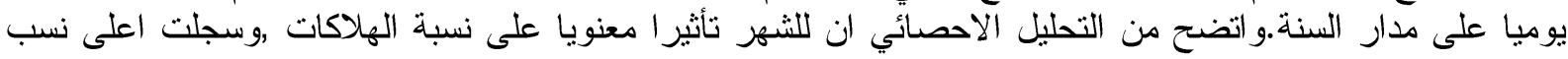
هلاكات في شهر ايار وحزيران وتموز وكانت اعلى نسبة هلاكات في شهر تموزحيث بلغت الته

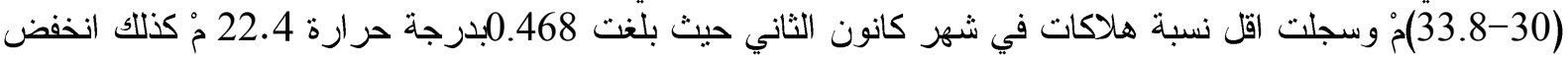

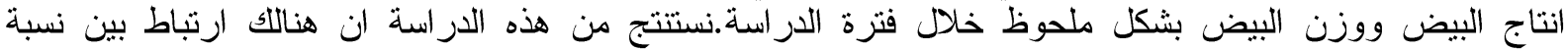

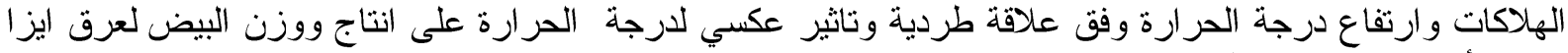
بر اون أي وجود تاثير للظروف المناخية و اضح على على كفاءة الاداء .

المقدمة الأماء

ووزن البيض وزيادة الهلاكات. تمنلك الطيور سبيلين للتخلص من الحرارة من الجسم بواسطة التبريد بدون

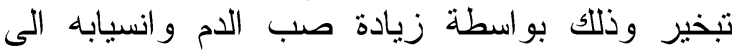

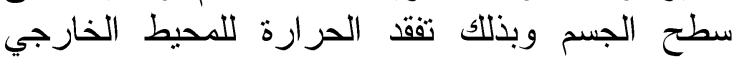

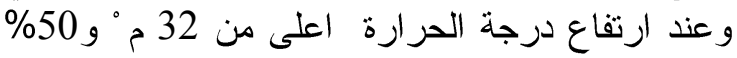

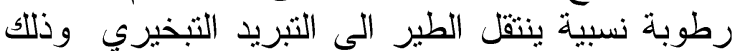

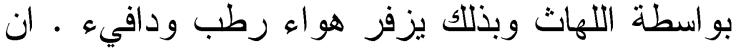
انحدار الحرارة بين المحيط وحرارة الجسم ضرولة ضرورية

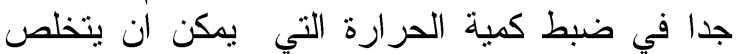

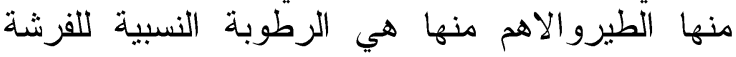

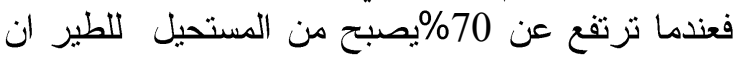

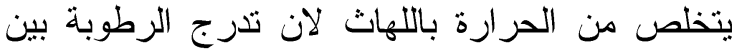

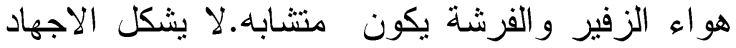

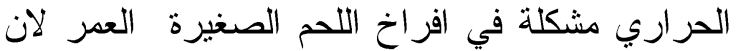

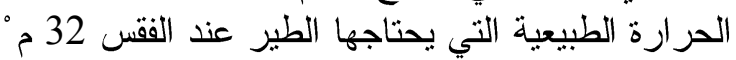

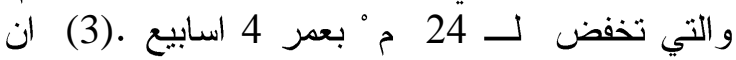
درجة حرارة الجسم الطبيعية للاجاج هي 41,4 لمن

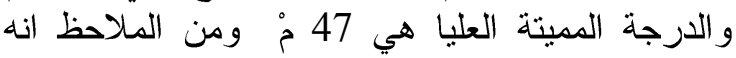

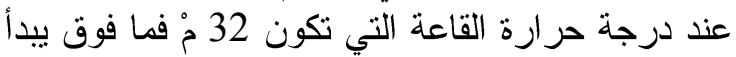

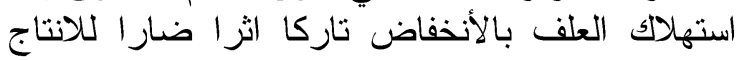

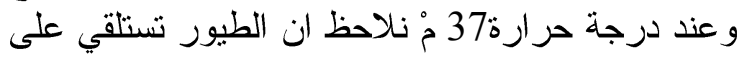

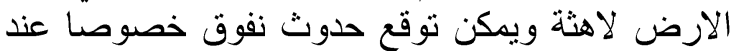

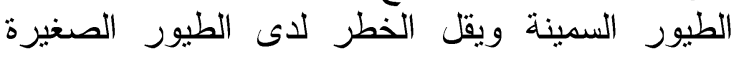

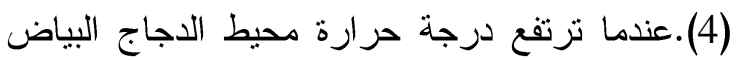

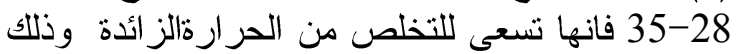

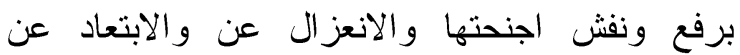
الطيور الاخرى و يكون فقدان الحرارة الملموسة الانعادئ

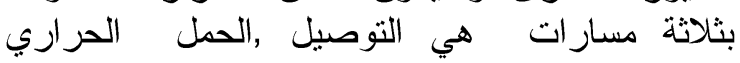

والإشعاع (5)
ان وجود تحديات الظروف الجويه على صناعة

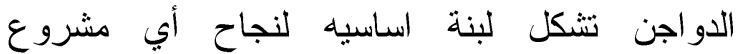

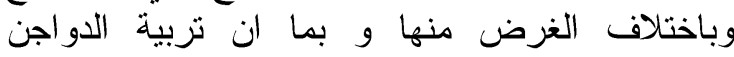

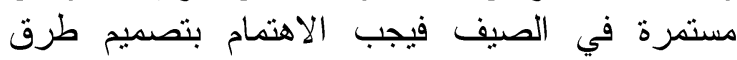

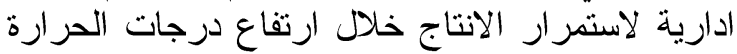

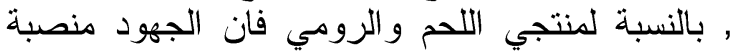

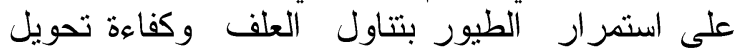

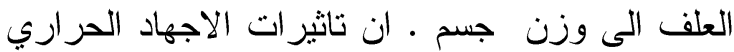

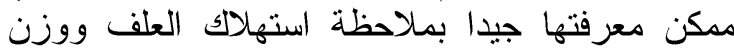

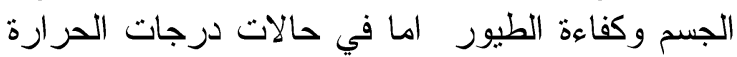

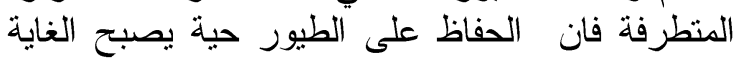

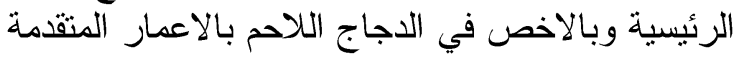

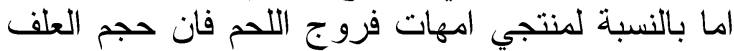

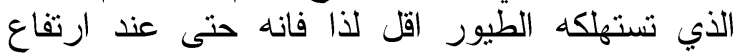

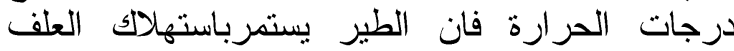

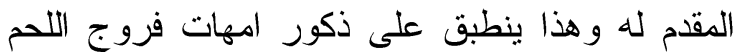

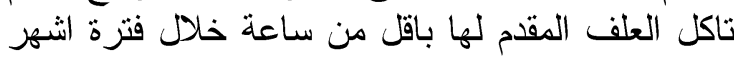

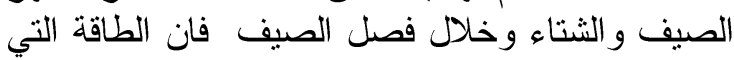

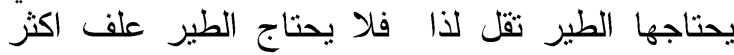

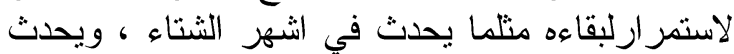

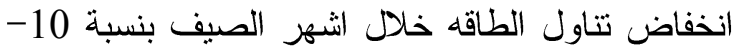

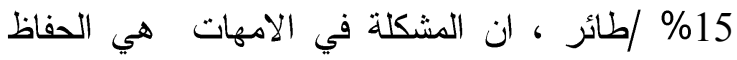

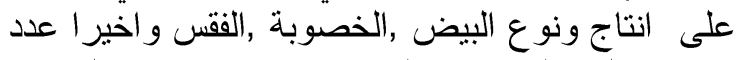

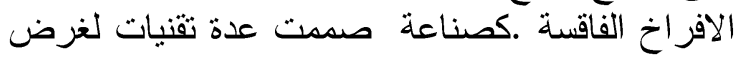

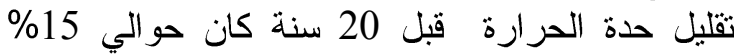

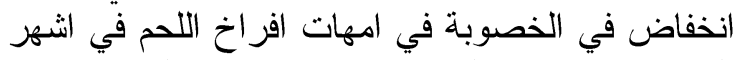

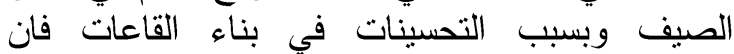

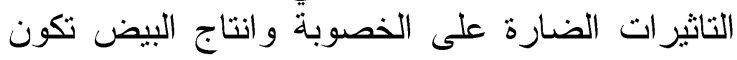

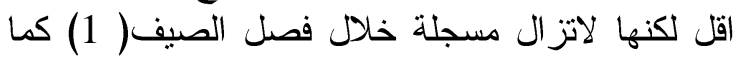

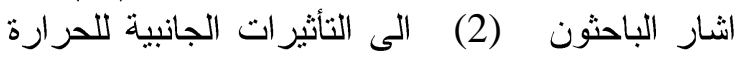
المرتفعة على الدجاج البياض من حيث انخفاض انتاج 


\section{المواد وطر ائق العمل}

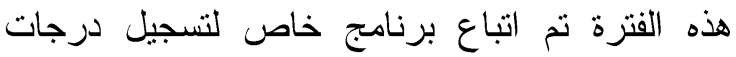

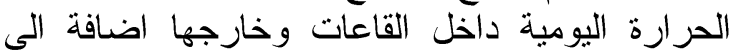

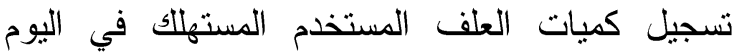
الواحد كما تم تسجيل كمية البيض المنتج يوميا ووزينه

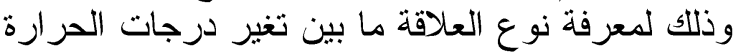

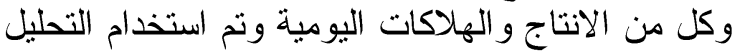
الاحصائي لايضاح نتائج التجربة.
استخدم في هذه الدراسة دجاج بياض سلالة

ايسا براون بعمر 122 اسبو ع في حقلين وكانت طاقية الحقل الواحد 5 قاعات ابعاد القاعة 98×13 13 م ر , العدد الكلي للاجاج في الحقل الاول كان 105030 اما الحقل العاعل

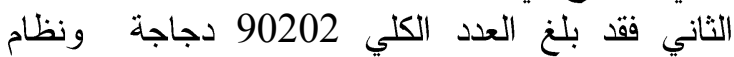
التربية المستخدم هو النظام العمودي لنظام التبريد المستخدم هو نظام اير ووشر و وقد تمت مر اقبة الحقلين

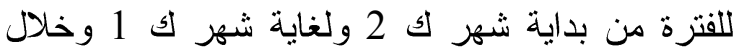
النتائج وخ

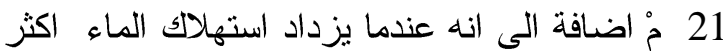
ت أنج ج والمناقشة

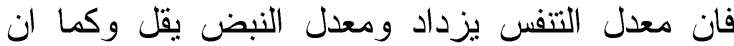

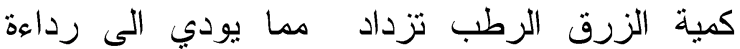

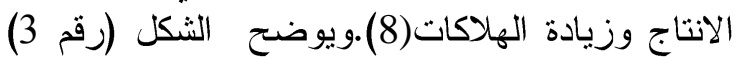

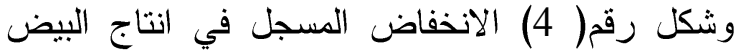

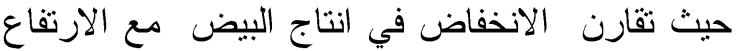

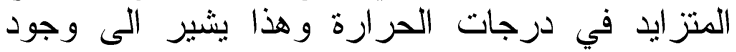

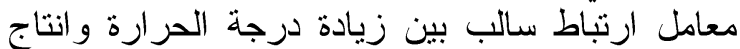

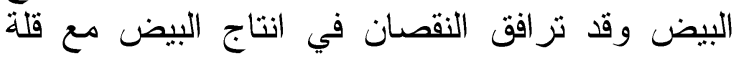

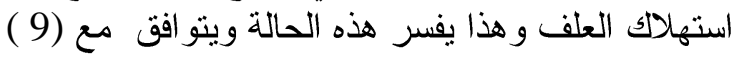

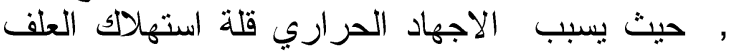

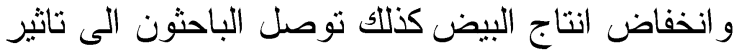

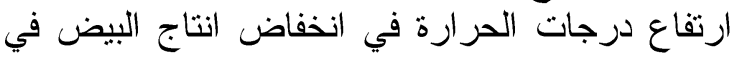

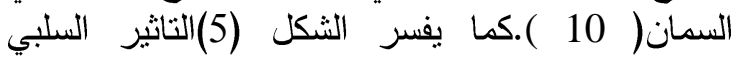

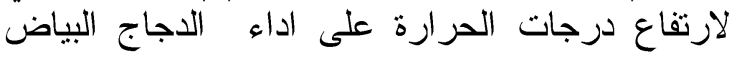

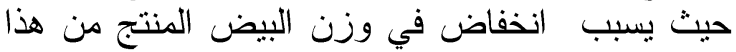

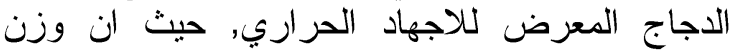

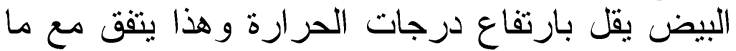

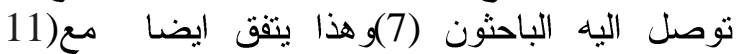

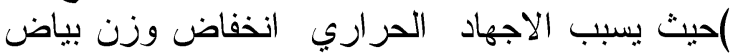

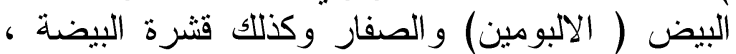

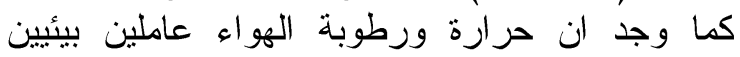

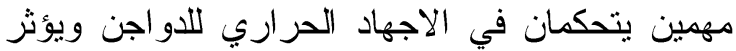

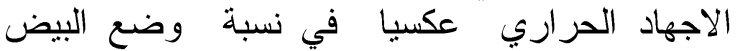

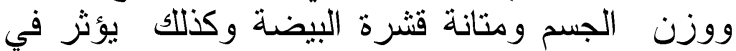

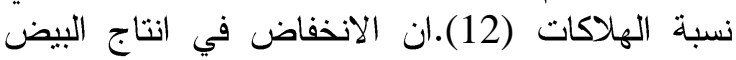

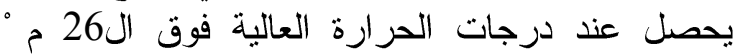

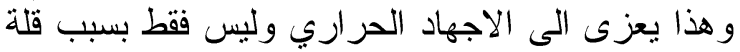

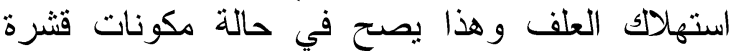
البيضة واختز ال ثخنها الذي يعود بالدرجة الاساس الى الى ارتفاع قلاء الدم alkalosis بسبب فقدان كميات كبيرة

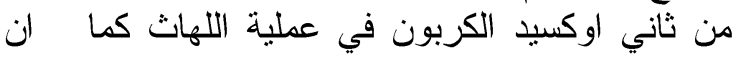
انخفاض وزن الالبومين والصفار يحدث ايضا بسبب



ان الاجهاد الحراري هو مشكلة تواجه وحدات الاتجات

انتاج الدواجن وحتى ذات الطاقة الانتاجية الكبيرة)

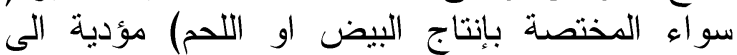

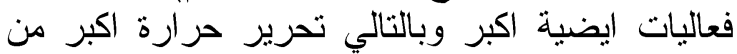

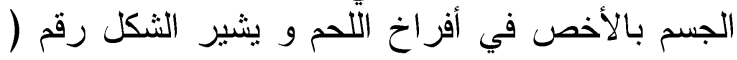

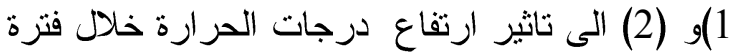

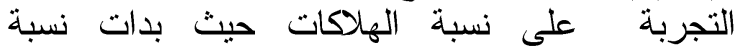
الهلاكات بالارتفاع ابتداءا من شهر مايس ولت وحزيران

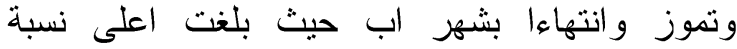
للهاكات في شهر تموز و هي

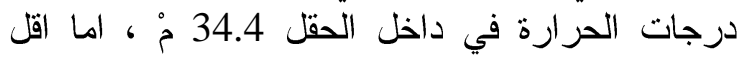
نسبة هلاكات فقد كانت في شهر فات فل في 2) فقد بلغت

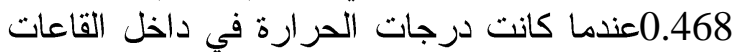

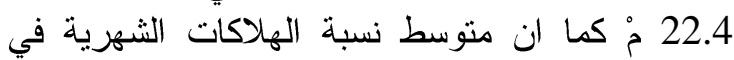

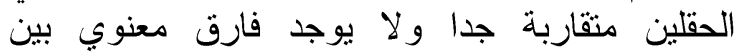

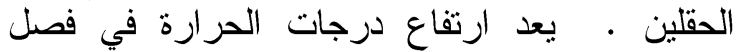

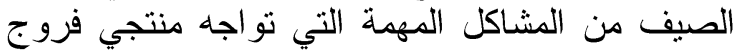

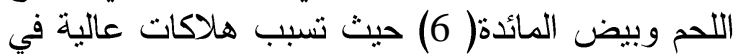

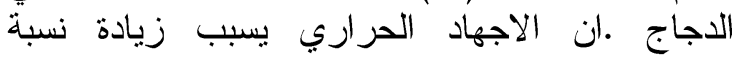

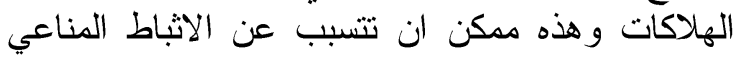

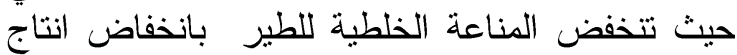

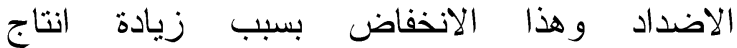
السايتوكاينيز الالتهابية تحت الاجهاد الحراري الإي والتي تحفز انتاج العامل المحرر للكورتكوتروبين من تحت العت العتادين

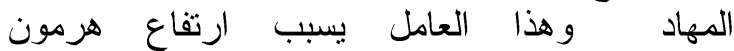

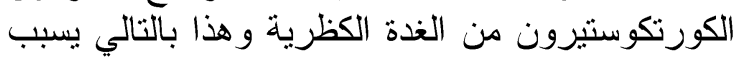

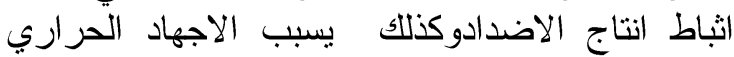
انخفاض الَسايتوكاينيز و الخلايا التائية المساعدة المهية

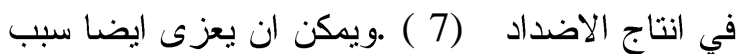

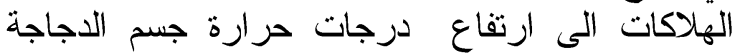

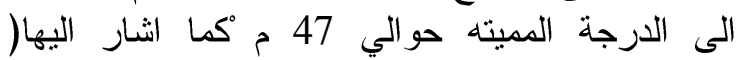
4)عندما يكون الجسم مجهز حراريا فان الطائر يميل الطيل للشرب اكثر ويقلل من الطعام وتقل الثهية بمقدار 1.5\% لكل درجة واحدة من الحرارة التي تزيد عن بلن 
مجلة القادسية لعلوم الطب البيطري العدد الخاص ببحوث المؤتمر العلمي الخامس 21-2012/11/22 المجلد 11 العبطري 32012

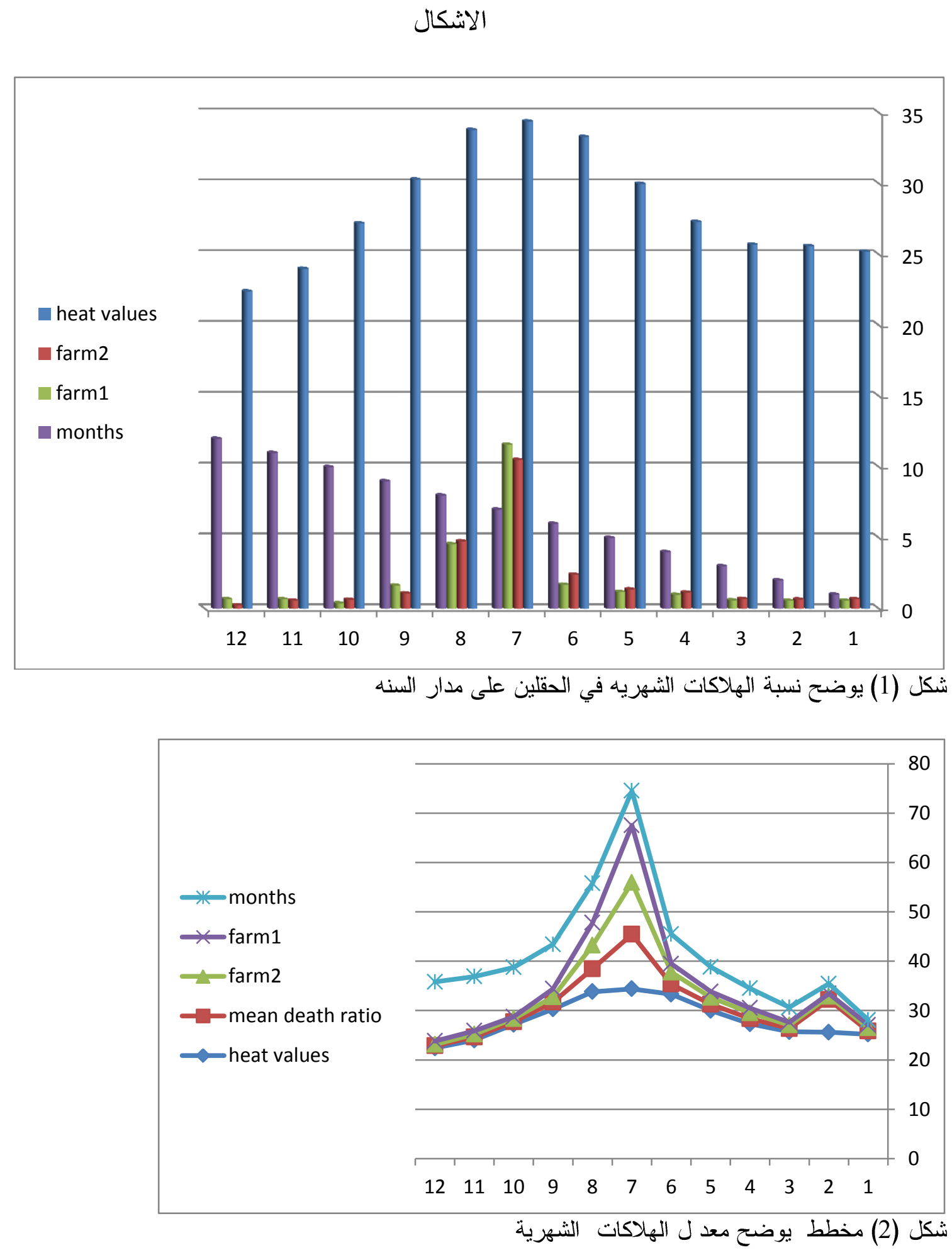


مجلة القادسية لعلوم الطب البيطري العدد الخاص ببحوث المؤتمر العلمي الخامس 21-2012/11/22 المجلد 11 البطري $2012 \quad 3$
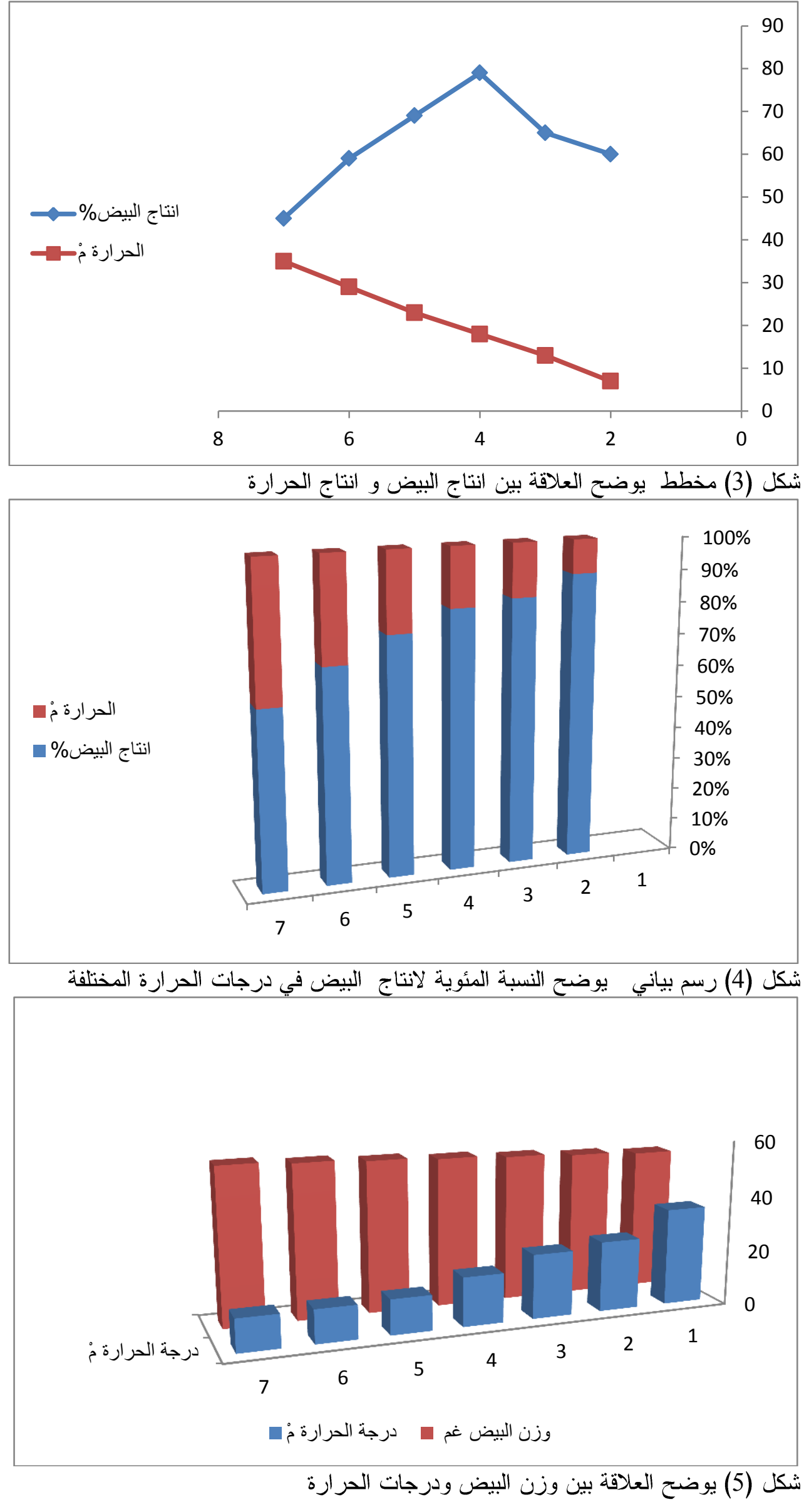


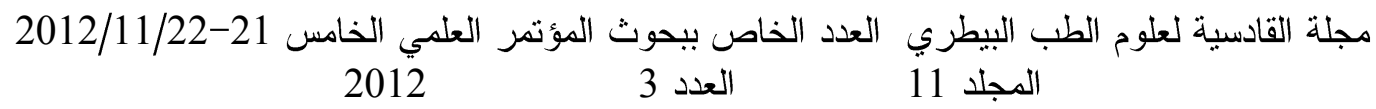

\section{| (لمصادر}

1. Bramwell,K. (2003). Effect Of Summer Heat Stress On Poultry Breeding Stock .Extension Poultry Specialist,Cooperative Extension Service, University Of Arkansas AVIAN Advice Newsletter, Avian Advice . 5, No.2.

2. Aberra, Melesse; S Maak; And G Von Lengerken(2010) Effect Of LongTerm Heat Stress On Egg Quality Traits Of Ethiopian Naked Neck Chickens And Their F1 Crosses With Lohmann White And New Hampshire Chicken Breeds . Livestock Research For Rural Development. Volume22,NO.4, ISSN 0121-3784

3. Teeter, Robert(1994) Heat Stress. Poultry Digest, May 1994, P10;In: Thepoultrysite. Com 5m Enterprises Ltd. 2-2000 - 2010 .

4. كماش,هاشم ناجي علي (1995) قطع الجناح واثره على بعض بعض الصفات الانتاجية و الفسلجية لفروج اللحم في ظروف ارتئن التفاع

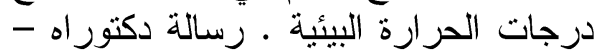
كلية الزر اعة جامعة بغداد.

5. Bird,N.A.; P. Hunton; W.D. Morrison And L.J. Weber(2011) Poultry: Heat Stress In Caged Layers Queen's Print. Ontario .

6. الزبيدي رصيب سعيد علوان(1986).ادارة الدو اجن.الطبعة الاولى

7. Abbas, A. O; M. M. Mashaly; G. L. Hendricks, ; M. A. Kalama; A. E. Gehad And P. H. Patterson(2004).Effect Of Heat Stress On Production Parameters And Immune Responses Of Commercial Laying Hens, Poultry Science 83:889-894.
8. Rural Chemical Industries (RCI) (Aust.) Pty Ltd.(2011).how to understand heat stress \& how to treat it

9. Deaton, J.W. (1983), Alleviation Of Heat Stress For Avian Egg Production -a Review. World's Poultry Science Journal 39: 210217 .Copyright Cambridge University Press 1983.

10. Aysöndü, M.H.; O. Özbey;N. Yildiz And Ö. Özmen. (2004).The Effects Of High Temperature On Blood Serum Parameters And The Egg Productivity Characteristics Of Japanese Quails (Coturnix Coturnix Japonica).International Journal Of Poultry Science 3 (7): 485-489

11. Ajakaiye ,Joachim J. ; Alcides PerezBelloand Angel MollinedaTrujillo (2011), Impact Of Heat Stress On Egg Quality In Layer Hens Supplemented With LAscorbic Acid And DlTocopherol Acetate .Veterinarski Arhiv 81 (1), 119-132.

12. Ajakaiye, Joachim J ; Joseph, O .A. And Sunday A .O..( 2010) Effects Of Heat Stress On Some Blood Parameters And Egg Production Of Shika Brown Layer Chickens Transported By Road. Biol Res 188 43, 2010, 183-189.

13. Smith, A . J. ( 1974 ).. Changes in the average weight and shell thickness of eggs produced by hens exposed to high environmental temperatures-A review. Tropical Animal Health and Production , Nov;6(4):237244. 
مجلة القادسية لعلوم الطب البيطري العدد الخاص ببحوث المؤتمر العلمي الخامس 21-2012/11/22 31 العدد 2012

\title{
A field study to demonstrate the impact of high temperatures in Al- Diwaniya province on ISA Brown
}

\author{
N. S. Jassim, \\ I. N. Abdullah \\ A. A. A. Abed \\ Coll. of Vet. Med. Unive.of Al-Qadissia
}

\begin{abstract}
This study aims to describe the impact of high temperatures in the tropics on laying hens ISA Brown and thus to reduce the economic losses caused by this effect.The present study was conducted on two fields from the fields of laying hens have been vaccinated according to a program for vaccines fixed did not get injured during the the experimental period was calculated morbidity daily throughout the year.It was evident from the statistical analysis that for the month of significant effect on the morbidity proportion, with the highest rates morbidity in the Oval test, June, July and was the highest morbidity in July amounted to $11.04 \%$ at a temperature $(30-33.8 \mathrm{~m})$ and recorded less morbidity rate in January as they hit 0.468 with a temperature $22.4 \mathrm{~m}$. we conclude from this study that there is a correlation between the percentage of morbidity high temperature according to the positive relationship , also it adversely effect the production \& weight of egg produced by such birds.
\end{abstract}

